\title{
CONTROL ÓPTIMO INVERSO PARA SISTEMAS NO LINEALES EN TIEMPO CONTINUO
}

\section{INVERSE OPTIMAL CONTROL FOR CONTINUOUS-TIME NONLINEAR SYSTEMS}

\author{
Carlos Vega-Pérez ${ }^{1}$ | Ricardo Alzate-Castaño²
}

Forma de citar: VEGA-PÉREZ Carlos, ALZATE-CASTAÑO Ricardo. Control óptimo inverso para sistemas no lineales en tiempo continuo. Respuestas. 2014; 19(1):13-18.

Recibido:

Noviembre 17 de 2013

Aceptado:

Enero 20 de 2014

\section{RESUMEN}

Antecedentes: La optimización aplicada al control automático permite obtener acciones de control que satisfacen no solo el objetivo de control, sino también la minimización de un determinado funcional de costo. Dinámicas complejas dificultan hallar la solución explícita de un problema de control óptimo. El control óptimo inverso evita la solución explícita de la ecuación de Hamilton-Jacobi-Bellman para determinar la ley óptima de control. Objetivo: Ilustrar la potencialidad del control óptimo inverso como alternativa para resolver problemas complejos de optimización en control. Métodos: Se describe el problema de control óptimo para motivar el control óptimo inverso. Se formulan resultados matemáticos generales y se ilustra su aplicación a través de casos de ejemplo. Resultados: Las formulaciones matemáticas presentadas son probadas analíticamente en casos del tipo óptimo cuadrático lineal (LQR) y óptimo inverso basado en funciones de control de Lyapuvov (LCF). Conclusión: Es posible formular un problema de control óptimo para sistemas de tipo no lineal, sin abordar la solución explícita del problema de optimización, mediante control óptimo inverso.

Palabras clave: Control óptimo, Control óptimo inverso, Ecuación de Hamilton-Jacobi-Bellman, Función de control de Lyapunov (CLF), Regulador cuadrático lineal (LQR).

\section{ABSTRACT}

${ }^{1}$ Ingeniero Electrónico carlos.vega1@correo.uis.edu.co Universidad Industrial de Santander Bucaramanga -Colombia

${ }^{2}$ Doctor en Automática ralzatec@uis.edu.co Universidad Industrial de Santander Bucaramanga -Colombia
Background: Optimization theory applied to automatic control allows governing actions reaching desired conditions but minimizing a given performance index. Such optimization tasks imply to solve complicated mathematical expressions. The inverse optimal control appears as alternative to find the optimal control law without the explicit solution for the Hamilton-Jacobi-Bellman equation. Objective: To show the potential of the inverse optimal control for solving complex optimization 
problems in control theory. Methods: A general description of the optimal control problem is performed, followed by the justification of an inverse optimal approach. Examples for illustration are properly selected. Results: Mathematical formulations given are applied to solve analytically the cases of a linear optimal quadratic regulator (LQR) and a nonlinear inverse optimal Lyapunov-based control problem (CLF). Conclusion: It is possible to solve optimal control problems for nonlinear systems, without explicitly facing the Hamilton-Jacobi-Bellman equation, by means of the inverse optimal control approach.

Keywords: Optimal control, Inverse optimal control, Hamilton-JacobiBellman equation, Control Lyapunov Function (CLF), Linear quadratic regulator (LQR).

\section{INTRODUCCIÓN}

E

control óptimo corresponde fundamentalmente con la aplicación de a optimización para realizar acciones de control, y a partir de ello, minimizar un funcional de costo asociado con variables del sistema como el error, la señal de control y el vector de estados. Los inicios de esta teoría del control óptimo pueden remontarse a los trabajos de Pontryagin [7] y Bellman [10], los cuales buscaban minimizar funcionales de costo a partir de una representación matemática de la energía del sistema a través de la denominada función hamiltoniana. Estos resultados significaban sin embargo, una alta complejidad al momento de encontrar soluciones analíticas cerradas para el problema de control, siendo incluso inexistentes en algunas ocasiones. Uno de los pocos resultados analíticos importantes de los cuales puede hacerse referencia en este caso, es el regulador óptimo lineal cuadrático (LQR) desarrollado por Kalman [11]. A partir de esto es bien conocida la dificultad para resolver la ecuación de Hamilton-JacobiBellman (HJB) que constituye la génesis del control óptimo. Los principales resultados obtenidos corresponden con aproximaciones numéricas que explotan las bondades de los sistemas de cómputo actuales, lo cual sin embargo no es viable para implementación de sistemas de control en tiempo real, como

ocurre por ejemplo en ambientes industriales. Como una alternativa, se propone en la literatura a partir de los trabajos realizados por Freeman and Kokotovic [12, 13], el problema de control óptimo inverso, es decir, dada una señal de control óptima estabilizante se requiere encontrar el funcional de costo que dicha señal minimiza. A través de este enfoque es posible resolver explícitamente la ecuación HJB mediante la definición de una función de control de Lyapunov con una forma predeterminada. Resultados recientes encontrados en la literatura al respecto del control óptimo inverso incluyen $[3,6,8,9$, 15]. El análisis de dicha técnica de control es por tanto el objetivo principal del presente artículo. Como contenidos se proponen: el problema de control óptimo en la sección 2; una revisión al problema de control óptimo inverso de tiempo continuo en la sección 3 y conclusiones en la sección 4.

\section{CONTROL ÓPTIMO}

Considere el siguiente sistema dinámico no lineal en tiempo continuo:

$$
\dot{x}(t)=f(x(t))+g(x(t)) u(t), \quad x_{o}=x(0),(1)
$$

donde $x(t) \in R^{n}$ es el vector de las variables de estado en función del tiempo; $u(t) \in R^{m}$ es el vector de entradas correspondiente con las señales de control, $f: R^{n} \rightarrow R^{n}$ y $g: R^{n} \rightarrow R^{n x m}$ son 
funciones, en general, no lineales del estado.

Para un problema de control óptimo, es preciso definir un funcional de costo o índice de desempeño $J(x(t), u(t)$ ) el cual depende de la trayectoria solución para (1) dada por $x(t)$ y el vector de control $u(t)$, que denotaremos simplemente $J$. El objetivo de este tipo de control es maximizar o minimizar, dependiendo de la necesidad del sistema, el índice de desempeño dado por (2):

$I=\mathcal{K}(x(T))+\int_{0}^{T} \mathcal{L}(x(t), u(t)) d t$,

donde $\mathcal{K}(x(T))$ y $\mathcal{L}(x(t), u(t))$ son funciones continuamente diferenciables con respecto a $x(t)$ y $\mathcal{L}(x(t), u(t))$ es continua con respecto a $u(t)$ [5]. El término $\mathcal{K}(x(T))$ es la contribución del estado final al funcional de costo mientras que el término $\int_{0}^{T} \mathcal{L}(x(t), u(t)) d t$ representa la contribución que se acumula con el tiempo.

Hay dos tipos principales de condiciones de optimalidad que garantizan la existencia, unicidad y estabilidad para una ley de control [2]: las condiciones necesarias de tipo Pontryagin (Principio del Máximo) y las condiciones de suficiencia de tipo Bellman (Programación Dinámica). La diferencia básica en estos dos enfoques es que el primero conduce a soluciones locales mientras el segundo conduce a soluciones globales (por medio de la solución de la ecuación en derivadas parciales mejor conocida como la ecuación de Hamilton-Jacobi- Bellman). Este último enfoque es más adecuado para el diseño de control en sistemas realimentados cuando el horizonte de tiempo es infinito [1] y será el que va a ser empleado a continuación para encontrar una ley de control $u(t)$ para el sistema.

La ley de control óptimo deberá satisfacer las siguientes condiciones [14]:

(i) Lograr una estabilidad asintótica (global) en el punto de equilibrio en el origen para el sistema (1). Esta condición implica que el sistema controlado resultante es estable y robusto ante la acción de perturbaciones. (ii) Minimizar el funcional de costo, definido en nuestro caso particular a partir de:

$I=\int_{0}^{\infty}\left(l(x(t))+u^{T}(t) R(x(t)) u(t)\right) d t$,

donde $\mathcal{K}(x(T))=0 \mathrm{y}$

$\mathcal{L}(x(t), u(t))=l(x(t))+u^{T}(t) R(x(t)) u(t)$, siendo a su vez $l(x(t)) \geq 0$, representando la ponderación del error y $R(x(t))>0$ para todo $x(t)$. Nótese también que a diferencia de (2), se define para este caso un horizonte infinito.

Cuando $I$ es un mínimo se llama la función de valor óptimo, la cual a su vez será considerada como una función de Lyapunov denotada por $\mathrm{V}(x)$.

De otro lado si $u(t)$ es óptima, se denotará como $u^{*}$. Para hallar esta ley de control óptima $u^{*}$ es necesario definir el hamiltoniano asociado al sistema. La función del hamiltoniano viene dada por la siguiente ecuación [2]:

$\mathcal{H}(x(t), u(t))=l(x(t))+u^{T}(t) R(x(t)) u(t)+$

$\left[\frac{\partial V}{\partial x}(f(x(t))+g(x(t)) u(t))\right]^{T}$

Para garantizar la optimalidad, se requiere que $\frac{\partial \mathcal{H}}{\partial u}=0$.

Por tanto, derivando la ecuación (4) con respecto a $u$ e igualando a cero se obtiene:

$\frac{\partial \mathcal{F}}{\partial u}=2 R(x(t)) u(t)+g^{T}(x(t)) \frac{\partial V}{\partial x}=0$.

A partir de lo cual es posible obtener como ley de control óptima a la expresión:

$u^{*}=-\frac{1}{2} R^{-1}(x(t)) g^{T}(x(t)) \frac{\partial V}{\partial x}$.

Lo anterior, se define de manera formal mediante el siguiente teorema [14]:

\section{Teorema 1: (Condición suficiente para la op- timalidad)}

Si existe una función semidefinida positiva $V(x)$ en $C^{1}$, que satisface la ecuación de HamiltonJacobi-Bellman (HJB):

$l(x)+\frac{\partial V}{\partial x} f(x)-\frac{1}{4} \frac{\partial V}{\partial x} g(x) R^{-1}(x) g^{T}(x) \frac{\partial V}{\partial x}=0$,

$V(0)=0$,
Enero - Junio 2014 ISSN 0122-820X PP: 13-18 
No. 1

Enero - Junio 2014 ISSN 0122-820X

PP: 13-18 donde $l(x)$ y $R^{-1}(x)$ vienen dados por el funcional de costo, mientras $f(x)$ y $g(x)$ por el sistema, entonces la ley de control:

$u^{*}=-k(x)=-\frac{1}{2} R^{-1}(x) g^{T}(x) \frac{\partial V}{\partial x}$,

garantiza una estabilidad asintótica (global) en el punto de equilibrio, y por tanto es el control óptimo de estabilización que minimiza el funcional de costo, garantizando $\lim _{t \rightarrow \infty} x(t)=0$ y siendo

\section{$V(x)$ la función de valor óptimo.}

Para resolver el problema de control óptimo anterior, es necesario hallar el funcional $V(x)$ el cual se puede obtener si se resuelve la ecuación (6). En general, es posible resolver dicho problema de control óptimo cuando se aplica al caso de los sistemas lineales invariantes en el tiempo, a través del denominado regulador cuadrático lineal (LQR) [1], un ejemplo del cual se ilustra a continuación:

- Ejemplo 1 (Ley de control óptima):

Para el siguiente sistema:

$\dot{x}(t)=x(t)+u, \quad x_{0}=x(0)$,

determinar la ley de control óptima que minimiza el funcional de costo:

$J=\int_{0}^{\infty}\left(x^{2}(t)+u^{2}(t)\right) d t$,

implica encontrar la solución a la ecuación de $x^{2}+x \frac{\partial V}{\partial x}-\frac{1}{4}\left(\frac{\partial V}{\partial x}\right)^{2}=0$, $V(0)=0$.

Por tanto, tras resolver la ecuación cuadrática en $\frac{\partial V}{\partial x}$ se obtiene: $\frac{\partial V}{\partial x}=2 x(1+\sqrt{2})$.
Ahora, integrando esta última expresión con respecto a $x$, se genera el funcional:

$V(x)=x^{2}(1+\sqrt{2})$,

a partir de lo cual, la ley de control toma la forma:

$u^{*}=-k(x)=-\frac{1}{2} \frac{\partial V}{\partial x}=-x(1+\sqrt{2})$.

De otro lado, para sistemas no lineales de la forma (1) la solución de la expresión (6) es una tarea muy compleja que incluso muchas veces no lleva a un resultado explícito, aún en el caso que esta exista. Para evitar este tipo de inconvenientes, se propone abordar la solución del problema de control óptimo propuesto a través del control óptimo inverso.

\section{CONTROL ÓPTIMO INVERSO PARA SEGUIMIENTO DE TRAYECTORIA}

El problema del control óptimo inverso surge como una alternativa para encontrar una ley de control óptima estabilizante en un sistema, sin la necesidad de resolver la correspondiente ecuación HJB.

Para este enfoque se establece a priori una ley de control para el problema y a posteriori se obtiene el funcional de costo con el conocimiento de la ley de control de realimentación estabilizante [14].

Inicialmente, se considera el error como $z=x-x_{d}$, donde $x_{d}$ son los valores deseados de $x$.

A continuación, se define la ley de control óptima inversa $u^{*}$ como:

$u^{*}=-k(z)=-\frac{1}{2} R^{-1}(z) g^{T}(z) \frac{\partial V}{\partial z}$

$R(x)>0$,

la cual será de estabilización inversa óptima a lo largo de la trayectoria deseada si se cumplen (i) y (ii). Para determinar esta ley de control óptima inversa se requiere el conocimiento de $V(z)$, por tanto esta última se establecerá como una función de control de Lyapunov (CLF) que nos permita garantizar el cumplimiento de las condiciones (i) y (ii). Una elección válida de $V(z)$ para este propósito corresponde con la función cuadrática:

$V(z)=\frac{1}{2} z^{T} P z, \quad P=P^{T}>0$,

la cual asegurará una estabilización global para el sistema, según se demuestra en [4]. Ahora bien, con base en esta suposición la ley de control optima inversa toma la siguiente forma:

$$
u^{*}=-\frac{1}{4} R^{-1}(z) g^{T}(z) \frac{\partial\left(z^{T} P z\right)}{\partial z}
$$




$$
u^{*}=-\frac{1}{2} R^{-1}(z) g^{T}(z) P z
$$

$u^{*}=-\frac{1}{2} R^{-1}(z) g^{T}(z) P\left(x-x_{d}\right)$,

donde $P=P^{T}>0$ y $R(z)=R(z)^{T}>0$ asegurando la existencia de las correspondientes matrices inversas.

A partir de la ley de control establecida en (10) se define el funcional de costo asociado a este problema de optimización por medio de la siguiente condición [14]:

Si existe una matriz P que cumpla la siguiente desigualdad:

$\dot{V}=\frac{\partial V}{\partial z} f(z)+\frac{1}{2} \frac{\partial V}{\partial z} g(z) u^{*} \leq 0, \quad u=\frac{1}{2} u^{*}$, $\dot{V}=\frac{\partial V}{\partial z} f(z)-\frac{1}{4} \frac{\partial V}{\partial z} g(z) R^{-1}(z) g^{T}(z) P z \leq 0, \quad$ (11)

se tiene que un funcional de costo expresado como en (2), es minimizado siempre que $l(z)$ sea de la forma:

$l(z):=-\dot{V}=-\frac{\partial V}{\partial z} f(z)+\frac{1}{4} \frac{\partial V}{\partial z} g(z) R^{-1}(z) g^{T}(z) P z \cdot$

En resumen, el problema de control óptimo inverso para seguimiento de trayectoria consiste en que a partir de la definición de la ley de control óptimo (10), la cual a su vez depende de la función $V(z)$ propuesta en (9), el funcional de costo (2) se minimiza siempre que $l(z)$ corresponda con (12). Lo anterior se ilustrará a partir del siguiente ejemplo.

- Ejemplo 2 (Ley de control óptima inversa):

Para el siguiente sistema:

$\dot{x}(t)=x(t)+x(t) u, \quad x_{o}=x(0)=10$,

determine empleando control óptimo inverso, el funcional de costo que minimiza la ley de control óptima (10).

Partiendo de la función de control de Lyapunov:

$V(z)=\frac{1}{2} z^{T} P z=5 z^{2}$,

donde $z=x-2$, la ley de control óptima inversa toma la forma:

$u^{*}=-\frac{10 x(x-2)}{2}, \quad R(z)=1$.

Ahora bien, considerando lo anterior, el término $l(z)$ se establece como:

$l(z):=-\dot{V}=-10 x(x-2)+25 x^{2}(x-2)^{2}$,

Enero - Junio 2014

ISSN 0122-820X

PP: $13-18$

a partir de lo cual el funcional de costo que minimiza esta función de control se define mediante:

$I=\int_{0}^{\infty}\left(-10 x(t)(x(t)-2)+25 x^{2}(t)(x(t)\right.$

$\left.-2)^{2}+u^{2}(t)\right) d t$

siendo la solución al problema de control óptimo inverso propuesto.

\section{AGRADECIMIENTOS}

Los autores desean agradecer la colaboración del Dr. Édgar Nelson Sánchez Camperos del CINVESTAV-Guadalajara en la discusión de los conceptos de base para los resultados analíticos presentados en este artículo y a la Vicerrectoría de Investigaciones de la Universidad Industrial de Santander VIEUIS por su apoyo mediante el programa de movilidad internacional de investigadores.

\section{CONCLUSIONES}

- Tras plantear y resolver la función hamiltoniana de un sistema dinámico, es posible encontrar la función de control $u(t)$ que minimiza un funcional de costo $J(x(t), u(t))$ determinado.

- En general la solución al problema de control óptimo a través de la ecuación de Hamilton-Jacobi-Bellman no es trivial ni presenta garantía de existencia.

- El control óptimo inverso aparece como una alternativa para determinar el funcional de costo minimizado por una ley óptima de control obtenida sin la necesidad de resolver la ecuación de Hamilton-Jacobi-Bellman.

- Se han presentado ejemplos para desarrollos de control óptimo tipo LQR y de control óptimo inverso basado en funciones de control de Lyapunov (CLF). 
- Actualmente se realiza la aplicación de los resultados genéricos presentados en este artículo, para manipular el comportamiento dinámico de circuitos convertidores de potencia en los laboratorios de Ingeniería Eléctrica de la Universidad Industrial de Santander (UIS) en Bucaramanga - Colombia.

\section{BIBLIOGRAFÍA}

[1]. B.D.O. Anderson, J.B. Moore, "Optimal Control: Linear Quadratic Methods". Prentice-Hall, Englewood,NJ, 1990.

[2]. D. E. Kirk, "Optimal Control Theory: An Introduction", Englewood Cliffs, NJ, USA: Prentice-Hall, 1970.

[3]. G.J. Ji. "Inverse Optimal Stabilization of a Class of Nonlinear Systems". Proceedings of the 26th Chinese Control Conference July 26-31, 2007, Hunan, 226-231.

[4]. J. J. Slotine, W. Li, "Applied Nonlinear Control", Massachusetts Institute of Technology, Englewood Cliffs, New Jersey, Prentice Hall.

[5]. Luenberger, D. G. "Introduction to dynamic systems Theory, Models and Applications", U.S.A, pp.419-427. 1979.

[6]. L. Rodrigues, "An Inverse Optimality Method to Solve a Class of Second Order Optimal Control Problems", IEEE 18th Mediterranean Conference on Control \& Automation, Marrakech, Morocco, pp.407-412, June 23-25, 2010.

[7]. L. S. Pontryagin, V. G. Boltyankii, R. V. Gamkrelizde, and E. F. Mischenko. "The Mathematical Theory of Optimal Processes." Interscience Publishers, Inc., New York, USA, 1962.

[8]. M. Krstic and Z. Li. "Inverse optimal design of input-to-state stabilizing nonlinear controllers". IEEE Transactions on Automatic Control, 43(3):336-350, 1998.

[9]. P. J. Moylan and B. D. O. Anderson. "Nonlinear regulator theory and an inverse optimal control problema". IEEE Transactions on Automatic Control, 18(5):460- 465, 1973.

[10]. R. E. Bellman. "Dynamic Programming”. Princeton, NJ, 1957.

[11]. R.E. Kalman, "Contributions to the theory of optimal control," Bol. Soc. Mat. Mex. vol. 5, pp. 102-119, 1960.

[12]. R. Freeman, P.V. Kokotovic. "Optimality of Robust Nonlinear Feedback Controls". Technical report CCEC-931103, 1993.

[13]. R. Freeman, P.V. Kokotovic, "Robust Control of Nonlinear Systems", Birkhauser, Boston, 1996.

[14]. R. Sepulchre, M. Jankovic, P.V. KokotoviC, "Constructive Nonlinear Control”, Springer, Berlin, 1996.

[15]. Z. Liu, Q. Wang, and H. Schurz, "Inverse optimal noise-to-state stabilization of stochastic recurrent neural networks driven by noise of unknown covariance," Optimal Control Applications and Methods, vol. 30, no. 2, pp. 163-178, 2009. 